\title{
African-American race and mortality in interstitial lung disease: a multicentre propensity-matched analysis
}

\author{
Ayodeji Adegunsoye ${ }^{1}$, Justin M. Oldham², Shashi K. Bellam ${ }^{3}$, \\ Jonathan H. Chung ${ }^{4}$, Paul A. Chung $\mathbb{1}^{5}$, Kathleen M. Biblowitz ${ }^{5}$, \\ Steven Montner ${ }^{4}$, Cathryn Lee ${ }^{1}$, Scully Hsu ${ }^{1}$, Aliya N. Husain ${ }^{6}$, Rekha Vij ${ }^{1}$, \\ Gokhan Mutlu' ${ }^{1}$, Imre Noth ${ }^{1}$, Matthew M. Churpek ${ }^{1,7,8}$ and Mary E. Strek ${ }^{1,8}$
}

Affiliations: ${ }^{1}$ Section of Pulmonary and Critical Care, Dept of Medicine, The University of Chicago, Chicago, IL, USA. ${ }^{2}$ Division of Pulmonary, Critical Care and Sleep Medicine, Dept of Medicine, University of California at Davis, Davis, CA, USA. ${ }^{3}$ Division of Pulmonary and Critical Care, Dept of Medicine, NorthShore University HealthSystem, Evanston, IL, USA. ${ }^{4}$ Dept of Radiology, The University of Chicago, Chicago, IL, USA. ${ }^{5}$ Dept of Medicine, NorthShore University HealthSystem, Evanston, IL, USA. ${ }^{6}$ Dept of Pathology, The University of Chicago, Chicago, IL, USA. ${ }^{7}$ Dept of Public Health Sciences, The University of Chicago, Chicago, IL, USA. ${ }^{8}$ Both authors contributed equally.

Correspondence: Ayodeji Adegunsoye, Section of Pulmonary and Critical Care, Dept of Medicine, The University of Chicago, 5841 S. Maryland Ave, Chicago, IL 60637, USA. E-mail: dejiduchicago.edu

@ERSpublications

African-American ILD subjects are younger, less often male and may have greater survival than other racial groups http://ow.ly/mvFQ30jOJAi

Cite this article as: Adegunsoye A, Oldham JM, Bellam SK, et al. African-American race and mortality in interstitial lung disease: a multicentre propensity-matched analysis. Eur Respir J 2018; 51: 1800255 [https:// doi.org/10.1183/13993003.00255-2018].

ABSTRACT We studied whether African-American race is associated with younger age and decreased survival time at diagnosis of interstitial lung disease (ILD).

We performed a multicentre, propensity score-matched analysis of patients with an ILD diagnosis followed at five US hospitals between 2006 and 2016. African-Americans were matched with patients of other races based on a time-dependent propensity score calculated from multiple patient, physiological, diagnostic and hospital characteristics. Multivariable logistic regression models were used. All-cause mortality and hospitalisations were compared between race-stratified patient cohorts with ILD, and sensitivity analyses were performed.

The study included 1640 patients with ILD, 13\% of whom were African-American, followed over 5041 person-years. When compared with patients of other races, African-Americans with ILD were younger at diagnosis ( 56 years versus 67 years), but in the propensity-matched analyses had greater survival (hazard ratio $0.46,95 \%$ CI $0.28-0.77 ; \mathrm{p}=0.003$ ) despite similar risk of respiratory hospitalisations (relative risk 1.04, 95\% CI 0.83-1.31; p=0.709), and similar GAP-ILD (gender-age-physiology-ILD) scores at study entry. Sensitivity analyses in a separate cohort of 9503 patients with code-based ILD diagnosis demonstrated a similar association of baseline demographic characteristics with all-cause mortality.

We conclude that African-Americans demonstrate a unique phenotype associated with younger age at ILD diagnosis and perhaps longer survival time. 


\section{Introduction}

The interstitial lung diseases (ILDs) are a heterogeneous group of diffuse parenchymal lung disorders with shared clinical, radiographic and pathologic features [1]. While some ILDs manifest with parenchymal inflammation at disease onset, others lead to fibrotic destruction. Such changes to the pulmonary parenchyma can lead to severe impairment of lung function and concomitant high mortality. While geographic differences in disease burden and variable recognition have led to substantial variation in the reported incidence of ILD, the global prevalence and mortality continue to rise with the ageing population worldwide [2,3].

Racial differences in disease risk and mortality are important determinants of population health and can result in health disparities $[4,5]$. African-Americans are the largest minority race in the United States, now encompassing $>40$ million people [6]. African-American race has been associated with high rates of respiratory impairment and worsened survival in numerous pulmonary disease conditions $[7,8]$. The US Centers for Disease Control and Prevention reports a three-fold increase in asthma-related deaths among African-Americans compared to European-Americans [6,9]. African-Americans have earlier onset of chronic obstructive pulmonary disease, worsened lung function decline and greater mortality risk with lung cancer when compared to European-Americans [10-12]. Likewise, the severity of connective tissue diseases (CTDs) and associated risk for pulmonary involvement is greater among African-Americans [13, 14].

The majority of epidemiological studies characterising patients with ILD and evaluating survival have been conducted in populations with a Caucasian predominance [15-18]. One such study suggested that patients of African descent are less likely to develop the most severe form of ILD, idiopathic pulmonary fibrosis (IPF), but that death from IPF occurs at a younger age in African-Americans [16]. However, it remains unclear whether racial differences influence survival across the wide spectrum of ILD. We anticipated that similar to other pulmonary disorders, earlier onset of ILD and worsened outcomes in African-Americans might prompt the need for earlier therapeutic intervention. Thus, we hypothesised that independent of access to care, African-American race is associated with younger age at ILD diagnosis and increased mortality when compared to other races.

\section{Methods}

\section{Study design and population}

This retrospective cohort study included all adult patients ( $\geqslant 18$ years) at five US hospitals with a multidisciplinary diagnosis of ILD (University of Chicago Hospital, Chicago, IL, USA) and independently adjudicated ILD diagnosis (Evanston Hospital, Evanston, IL; Highland Park Hospital, Highland Park, IL; Glenbrook Hospital, Glenview, IL; and Skokie Hospital, Skokie, IL) from January 2006 through July 2016. The electronic medical record, which contained inpatient and outpatient data, was used to ascertain supportive evidence of ILD and race category and to determine vital status. The categorisation of race was implemented per the federally defined US Census Bureau standards on race (White, Black or African-American, American-Indian or Alaska Native, Asian and Native Hawaiian or other Pacific Islander) and ethnicity (Hispanic or not Hispanic) [19]. No individuals identified as American-Indian or Native Hawaiian in the study cohort. The remaining categories were stratified into African-American (non-Hispanic black) and non-African-American (non-Hispanic white, non-Hispanic Asian and Hispanic) cohorts. The study was reviewed and approved by the local institutional review boards (16-1062 and 17-025), which waived informed consent.

\section{Multidisciplinary diagnosis of ILD}

To assess our findings, patients with International Statistical Classification of Diseases, 9th revision (ICD-9) code-based ILD diagnosis at the tertiary hospital (University of Chicago Hospital) underwent a multidisciplinary evaluation using available clinical data, pulmonary function tests (PFTs), high-resolution computed tomography (HRCT) scans and surgical lung biopsies according to current American Thoracic Society/European Respiratory Society criteria. An assessment of multidisciplinary diagnosis (MDD) of ILD was performed by pulmonologists in conjunction with rheumatologists, dedicated chest radiologists and a thoracic pathologist. Patients with an eventual multidisciplinary diagnosis of sarcoidosis were excluded, as the majority of patients with pulmonary sarcoidosis lack parenchymal lung disease. Ascertainment of race was performed by separate reassessment of clinician-documented race at the time of PFTs.

As the four non-tertiary hospitals are suburban community hospitals that do not perform multidisciplinary discussions to determine ILD diagnosis, an independent "adjudication" panel of two academic pulmonologists with expertise in ILD ( JMO and RV) evaluated clinical data including PFT results and HRCT scans to confirm the diagnosis of ILD in all patients who received an ICD-9 code-based ILD diagnosis from a pulmonologist at that centre, and had clinical information available for review. All adjudicators were blinded to race, hospitalisation and mortality data. Each panel member reviewed all available records to determine whether the study participant had ILD. Among discordant cases $(n=60)$, a third independent pulmonologist 
with ILD expertise reviewed the available data for eventual classification. Based on review of this information, patients classified as having ILD $(n=488)$ were included in the analyses (agreement $=92.6 \%, \kappa=0.84$ for ILD diagnosis). An ILD diagnosis was adjudicated by panel members when the available medical records that were reviewed demonstrated pulmonologist-diagnosed ILD with 1) radiographic evidence of pulmonary fibrosis; 2) HRCT ground-glass opacities with restrictive pulmonary defect; or 3) auscultatory crackles with restrictive pulmonary defect with or without impairment of diffusing capacity of the lung for carbon monoxide (DLCO) or oxygen requirement (online supplementary table E1).

\section{Propensity score matching}

To minimise potential confounding, African-Americans were matched to non-African-Americans using propensity score matching by calculating the probability of identification as African-American, given the study covariates on cohort entry. We selected covariate predictors for race in our propensity score based on previously identified socioeconomic factors associated with race and mortality in similar investigations that evaluated the influence of African-American race on mortality [20-22]. These were factors that had a plausible direct or indirect relation to both race and mortality. Covariates for assessing the primary outcome of the study included demographic characteristics, ILD subtype and pulmonary function measures of disease severity. Random frequency matching using the propensity score was performed, which increased the likelihood that all matches were of equal quality. The final cohort consisted of $~ 3: 2$ frequency-matched participants of African-American race to non-African-Americans.

\section{Follow-up and study outcomes}

Patients entered the cohort on the date of initial ILD evaluation at the centre. For the primary outcome, all patients were followed-up until occurrence of death, lung transplantation, end of the study period or loss to follow-up. Person-time was averaged at 30 days per month from initial ILD evaluation to study end-point. The primary outcome was all-cause mortality during the study follow-up period, and secondary outcomes included all-cause hospitalisations and respiratory hospitalisations. Additionally, survival was evaluated by constructing transplant-free, transplant-excluded and transplant-as-a-competing-risk-event Cox regression models (data not shown). The GAP (gender-age-physiology)-ILD score was assessed at initial presentation as an index of clinical disease severity [23].

\section{Mortality ascertainment}

Manual review of hospital documentation, death occurrence records and institutional review board-approved telephone confirmation was performed for ascertainment of vital status in cases of loss to follow-up. Vital status was confirmed using medical records and social security death index.

\section{Statistical analyses}

In the study cohort, hypothesis testing was conducted between pairs of variables using two-tailed t-tests for continuous variables and Fisher exact tests for categorical variables. All-cause and respiratory hospitalisation risks were computed using Poisson regression models.

A propensity score was generated for each patient using the "teffects" package in Stata [24, 25] by constructing a parsimonious unconditional logistic regression model that adjusted for potential confounding variables for ILD disease severity and mortality. Final model selection was based on the assessment of optimal covariate balance by comparing the standardised differences between groups after the propensity score was computed. The final model chosen adjusted for age, sex, body mass index, smoking status (ever versus never), forced vital capacity (FVC) \% predicted, DLCO \% pred, ILD subtype and hospital centre. We excluded referral status, income level and insurance status from the propensity score due to poor covariate balance, but did include these variables along with immunosuppressive therapy in subsequent outcome modelling. Patient selection for outcome modelling was performed using the propensity score with robust estimates of the variance-covariance matrix to calculate the $95 \%$ confidence interval level. We tested fully adjusted models for effect modification by the individual components of the GAP-ILD score (age, sex, FVC \% pred, DLCO \% pred and ILD subtype) using multiplicative interaction terms. Outcome modelling included random-effects analyses to control for heterogeneity between hospital centres. Patient stratification by centre was performed to assess consistency of study results across centres. Post-estimation tests demonstrated goodness of fit for all models.

Time to all-cause mortality was analysed using Cox proportional hazards models with robust standard errors to account for familial correlation in our cohorts. Survival time was calculated as time from initial ILD evaluation to death, lung transplantation, loss to follow-up or end of study period. Survival time was censored on July 31, 2016 or at the time a patient underwent lung transplantation or was lost to follow-up. Survival curves are plotted using the Kaplan-Meier survival estimator. Hazard ratios and odds ratios are reported relative to study participants of non-African-American race. 
Sensitivity analyses were performed in patients with code-based ILD diagnosis that did not meet the initial inclusion criteria of MDD-ILD (University of Chicago Hospital) or independently adjudicated ILD diagnosis (Evanston Hospital, Highland Park Hospital, Glenbrook Hospital and Skokie Hospital). Additional sensitivity analyses assessing sub-populations of the MDD-ILD and adjudicated ILD cohorts for the primary outcome were conducted. All p-values were two-sided and a level of 0.05 was considered statistically significant. All statistical analyses were performed in Stata (StataCorp 2017; R.15, StataCorp, College Station, TX, USA).

\section{Results}

Of the 1823338 patients evaluated across all study centres between January 2006 and July 2016, 11143 adult patients had an ICD-9 diagnosis code for ILD (online supplementary figure E1). Among these, 1640 patients had MDD or independently adjudicated ILD and were evaluated during 5041 person-years of follow-up. All adult age groups were represented (median (interquartile range) age 68 (59-75) years), and $821(50 \%)$ were male (table 1$)$.

TABLE 1 Baseline characteristics and demographics of the population with interstitial lung disease (ILD) diagnosis"

\begin{tabular}{|c|c|c|c|c|c|}
\hline & \multicolumn{4}{|c|}{ Pooled population } & \multirow[t]{3}{*}{ p-value ${ }^{\text {I }}$} \\
\hline & \multirow{2}{*}{$\begin{array}{l}\text { African-American patients } \\
\text { Black }\end{array}$} & \multicolumn{3}{|c|}{ Non-African-American patients } & \\
\hline & & White & Hispanic & Asian & \\
\hline Patients n & 222 & 1251 & 92 & 75 & \\
\hline Age years & $57.5 \pm 13.2$ & $68.4 \pm 11.5$ & $62.7 \pm 12.7$ & $64.3 \pm 14.9$ & $<0.001$ \\
\hline Male & $56(25.2)$ & $687(54.9)$ & $42(45.7)$ & $36(48.0)$ & $<0.001$ \\
\hline Ever-smoker & 97 (43.7) & $579(46.3)$ & $40(43.5)$ & $15(20.0)$ & 0.777 \\
\hline BMI $\mathrm{kg} \cdot \mathrm{m}^{-2}$ & $30.5 \pm 7.5$ & $29.4 \pm 6.4$ & $30.0 \pm 5.4$ & $26.2 \pm 6.2$ & 0.014 \\
\hline \multicolumn{6}{|l|}{ Clinical features } \\
\hline Crackles $^{+}$ & $137(71.7)$ & $802(80.4)$ & 67 (81.7) & 44 (75.9) & 0.532 \\
\hline Clubbing $^{+}$ & $22(9.9)$ & $147(11.8)$ & $13(14.1)$ & $8(10.7)$ & 0.400 \\
\hline Positive ANA titre ${ }^{\S}$ & $121(59.6)$ & $378(43.3)$ & $37(52.1)$ & $16(40.0)$ & $<0.001$ \\
\hline \multicolumn{6}{|l|}{ Comorbid diseases $^{+}$} \\
\hline Coronary artery disease & 13 (7.3) & $269(25.5)$ & $13(16.7)$ & 12 (18.2) & $<0.001$ \\
\hline Diabetes & $38(21.4)$ & $181(17.1)$ & 19 (24.4) & $20(30.3)$ & 0.331 \\
\hline Gastro-oesophageal reflux & $86(40.2)$ & $521(43.7)$ & 39 (44.3) & 25 (34.3) & 0.407 \\
\hline \multicolumn{6}{|l|}{ Lung function ${ }^{+}$} \\
\hline TLC \% & $64.6 \pm 16.2$ & $73.4 \pm 17.8$ & $64.7 \pm 14.0$ & $66.7 \pm 18.4$ & $<0.001$ \\
\hline FVC $\%$ & $56.8 \pm 16.2$ & $69.4 \pm 18.8$ & $61.5 \pm 17.8$ & $61.1 \pm 20.1$ & $<0.001$ \\
\hline$D$ LCO $\%$ & $46.1 \pm 18.5$ & $50.7 \pm 21.6$ & $48.2 \pm 21.6$ & $44.2 \pm 17.3$ & 0.016 \\
\hline 6MWD foot & $1007 \pm 365$ & $1114 \pm 407$ & $1110 \pm 309$ & $973 \pm 406$ & 0.009 \\
\hline HRCT fibrosis ${ }^{+}$ & $163(78.4)$ & 941 (82.8) & 64 (86.5) & 50 (73.5) & 0.082 \\
\hline Reticulation & $135(64.9)$ & $781(68.8)$ & 57 (77.0) & 38 (55.9) & 0.036 \\
\hline Traction bronchiectasis & $50(24.0)$ & $408(35.9)$ & 30 (40.5) & $25(36.8)$ & 0.006 \\
\hline Honeycomb pattern fibrosis & $75(36.1)$ & $438(38.6)$ & 35 (47.3) & $24(35.3)$ & 0.356 \\
\hline \multicolumn{6}{|l|}{ ILD sub-category } \\
\hline IPF & $28(12.6)$ & 555 (44.4) & 38 (41.3) & $33(44.0)$ & $<0.001$ \\
\hline $\mathrm{CHP}$ & $8(3.6)$ & $123(9.8)$ & $13(14.1)$ & $3(4.0)$ & 0.003 \\
\hline IPAF & 34 (15.3) & $129(10.3)$ & $10(10.9)$ & $8(10.7)$ & 0.029 \\
\hline CTD-ILD & $104(46.9)$ & $134(10.7)$ & 20 (21.7) & $7(9.3)$ & $<0.001$ \\
\hline Unclassifiable/other & $48(21.6)$ & $307(24.5)$ & $11(12.0)$ & $23(30.7)$ & 0.429 \\
\hline Oxygen therapy & $66(29.7)$ & $398(31.8)$ & 25 (27.2) & 19 (25.3) & 0.661 \\
\hline Immunosuppressive therapy & $120(55.8)$ & $563(46.4)$ & $46(50.6)$ & $42(57.5)$ & 0.020 \\
\hline Antifibrotic therapy & $4(1.8)$ & $101(8.1)$ & $5(5.4)$ & $3(4.0)$ & 0.001 \\
\hline
\end{tabular}

Data are presented as mean \pm SD or $n(\%)$, unless otherwise stated. $n=1640$. BMI: body mass index; ANA: antinuclear antibody; TLC: total lung capacity; FVC: forced vital capacity; DLCo: diffusing capacity of the lung for carbon monoxide; 6MWD: 6-min walking distance; HRCT: high-resolution computed tomography; IPF: idiopathic pulmonary fibrosis; CHP: chronic hypersensitivity pneumonitis; IPAF: interstitial pneumonia with autoimmune features; CTD: connective tissue disease. ${ }^{\#}$ : no individuals identified as American-Indian or Native Hawaiian in the study; ": comparing African-Americans to non-African-American categories, as follows. Pooled population $n=1640$; tertiary medical centre $n=1152$ (African-American $n=195$, non-African-American $\mathrm{n}=957$ ); non-tertiary medical centre $\mathrm{n}=488$ (African-American $\mathrm{n}=27$, non-African-American $n=461) ;{ }^{+}$: exceptions for count variables: crackles $n=1329$, clubbing $n=1369$, coronary artery disease $n=1380$, diabetes $n=1380$, gastro-oesophageal reflux $n=1568$, TLC $n=1271$, FVC $\mathrm{n}=1504, D$ LCO $\mathrm{n}=1425,6$ MWD $\mathrm{n}=793$, HRCT $\mathrm{n}=1508$; ${ }^{\text {}}$ : positive ANA titre $>1: 320$. 
Prevalence and baseline characteristics of African-Americans

Overall, there were 222 (13.5\%) patients that identified as African-American during the study period (table 1). Compared to the non-African-American population, the African-American population differed with regard to baseline characteristics, lung function and ILD sub-category (table 1). African-American patients were younger than non-African-Americans (58 years versus 68 years), had the lowest male prevalence $(25 \%)$, the greatest prevalence of positive antinuclear antibody titres $(60 \%)$ and the lowest prevalence of coronary artery disease $(7 \%)(\mathrm{p}<0.001)$ (table 1 ; figure 1a). In addition, African-Americans had substantially decreased measures of lung function: total lung capacity (TLC) 65\%, FVC 57\%, DLCO $46 \%$ and 6-min walking distance (6MWD) 1007 foot; and the least prevalence of traction bronchiectasis (24\%) ( $\mathrm{p}=0.006) \quad$ (table 1). When socioeconomic factors were assessed, in comparison to non-African-Americans, the majority of African-American patients had incomes below the national median $(72.9 \%$ versus $35.5 \%, \mathrm{p}<0.001)$ and fewer African-Americans were referred from out of state ( $10.4 \%$ versus $15.6 \%, \mathrm{p}=0.042$ ). African-Americans had greater odds of having CTD-ILD (OR 6.28, 95\% CI 4.32-9.12; $\mathrm{p}<0.001$ ) or interstitial pneumonia with autoimmune features (OR 1.66, 95\% CI 1.07-2.58; $\mathrm{p}=0.023$ ), but decreased odds of IPF (OR $0.21,95 \%$ CI $0.13-0.32 ; \mathrm{p}<0.001$ ) or chronic hypersensitivity pneumonitis (OR $0.28,95 \%$ CI $0.12-0.62$; p<0.001) (online supplementary table E2).

\section{Mortality in African-Americans with ILD}

In African-Americans with ILD, annual mortality rates increased with increasing age, peaking in the seventh decade of life during the study period (online supplementary figure E2). When assessing the primary outcome, survival of African-American patients was greater than that of non-African-Americans with ILD (figure 1).

The mean survival time among African-American patients was 103 months (95\% CI 96-110 months) compared to 85 months (95\% CI 81-88 months) in non-African-Americans. Mortality was $19 \%(42$ deaths) in African-Americans compared to 27\% (377 deaths) in non-African-Americans over the 10-year study period. Lung transplantation occurred in $5 \%(n=10)$ of African-American patients versus $4 \%(n=55)$ of non-African-American patients (table 2).

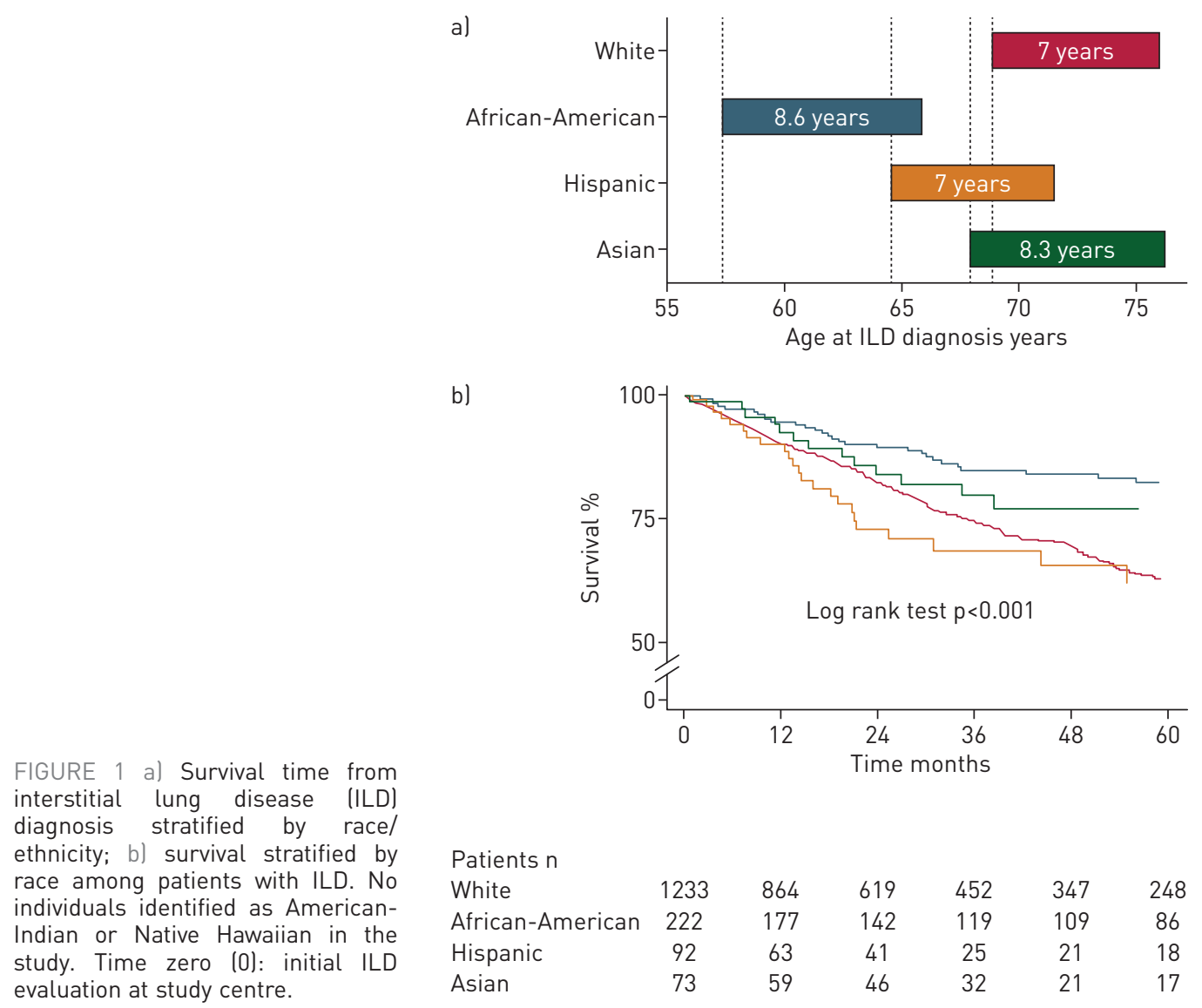


TABLE 2 Association between African-American race, mortality, all-cause hospitalisations and respiratory hospitalisations

\begin{tabular}{|c|c|c|c|}
\hline & African-American patients & Non-African-American patients & p-value \\
\hline Patients & 222 & 1418 & \\
\hline \multicolumn{4}{|l|}{ Mortality } \\
\hline Mortality & $42(18.9)$ & $377(26.6)$ & 0.015 \\
\hline Absolute mortality rate (events per person-year) & 0.04 & 0.09 & $<0.001$ \\
\hline Relative mortality risk ${ }^{\#}$ (unadjusted) & 0.51 & & $<0.001$ \\
\hline \multicolumn{4}{|l|}{ All-cause hospitalisations } \\
\hline Hospitalisation count (n per hospitalised patient) & $336(2.9)$ & $1785(3.1)$ & 0.588 \\
\hline Hospitalisation rate (events per person-year) & 0.35 & 0.44 & $<0.001$ \\
\hline Relative risk of hospitalisation ${ }^{+}$(unadjusted) & 0.81 & & $<0.001$ \\
\hline Relative risk of hospitalisation ${ }^{+}$(adjusted ${ }^{\text {I) }}$ & 0.78 & & $<0.001$ \\
\hline \multicolumn{4}{|l|}{ Respiratory hospitalisations } \\
\hline GAP-ILD score median (interquartile range) & $4(1-8)$ & $4(0-8)$ & 0.511 \\
\hline \multicolumn{4}{|c|}{$\begin{array}{l}\text { Data are presented as } \mathrm{n} \text { or } \mathrm{n}(\%) \text {, unless otherwise stated. GAP: gender-age-physiology; ILD: interstitial lung disease. }{ }^{\#} \text { : computed using Cox } \\
\text { proportional hazard models; }{ }^{\uparrow} \text { : adjusted for baseline demographic characteristics: body mass index, age, sex, forced vital capacity \% predicted, } \\
\text { diffusing capacity of the lung for carbon monoxide \% predicted, ILD subtype, GAP-ILD score, immunosuppressive therapy, antifibrotic therapy } \\
\text { and socioeconomic determinants of access to care; }{ }^{+} \text {: computed using Poisson regression models. }\end{array}$} \\
\hline
\end{tabular}

Before propensity score matching, the univariate and multivariable Cox hazards for all-cause mortality were decreased in African-American patients (HR 0.5, 95\% CI 0.4-0.7; p<0.001 and HR 0.4, 95\% CI $0.3-$ $0.6 ; \mathrm{p}<0.001$, respectively) (table 2; figure 2a). This decreased mortality was also observed in the code-based ILD subset without multidisciplinary or adjudicated ILD (HR 0.7, 95\% CI 0.7-0.8; p<0.001) (figure $2 \mathrm{~b}$ ). When specific ILD subtypes were examined, African-American race was associated with a consistent trend to improved mortality except in patients with IPF (figure $2 \mathrm{c}-\mathrm{f}$ ).

After propensity score matching, the unadjusted propensity score model constructed for the MDD and independently adjudicated ILD cohort included 306 patients (table 3). In primary outcome analysis of the unadjusted propensity score model, African-Americans had improved survival when compared with patients of other races (HR 0.51, 95\% CI 0.32-0.80; p=0.004) (table 4; figure 3). The improvement in survival remained consistent after adjusting for baseline demographic characteristics, physiological indices of disease severity using the GAP-ILD score, ILD subtype, referral status, use of immunosuppressive therapy, income level and type of insurance (HR 0.46, 95\% CI 0.28-0.77; p=0.003) (table 4).

Sensitivity analyses that assessed the baseline characteristics of the African-American population and tested study assumptions were performed in those patients with only a code-based ILD diagnosis with consistent results to those from the primary analysis (online supplementary table E3). Additional sensitivity analyses were performed in patients with MDD or adjudicated-ILD diagnosis adjusting for antifibrotic therapy, antinuclear antibody seropositivity, TLC, 6MWD and honeycomb fibrosis on HRCT yielded similar findings consistent at both tertiary and non-tertiary medical centres, and yielded similar results even after sub-stratifying by age, sex, GAP-ILD score and presence or absence of honeycombing (online supplementary table E4; online supplementary figure E3a-c). Although missing covariates were infrequent in our cohort $(<5 \%)$, additional data analysis accounting for these covariate values with multiple imputation using chained equations yielded similar results (data not shown).

\section{All-cause hospitalisation}

Among patients with MDD or adjudicated ILD, 40.6\% ( $n=692)$ were hospitalised during the study period. African-American patients had decreased risk for all-cause hospitalisation (relative risk (RR) $0.81,95 \% \mathrm{CI}$ 0.72-0.91; $\mathrm{p}<0.001$ ), when compared to non-African-American patients (table 2). This decreased risk remained consistent even after adjusting for baseline demographic characteristics, physiological indices of disease severity using the GAP-ILD score, ILD subtype, referral status, use of immunosuppressive therapy, income level and type of insurance (RR 0.78, 95\% CI 0.69-0.90; $\mathrm{p}<0.001)$ (table 2). 


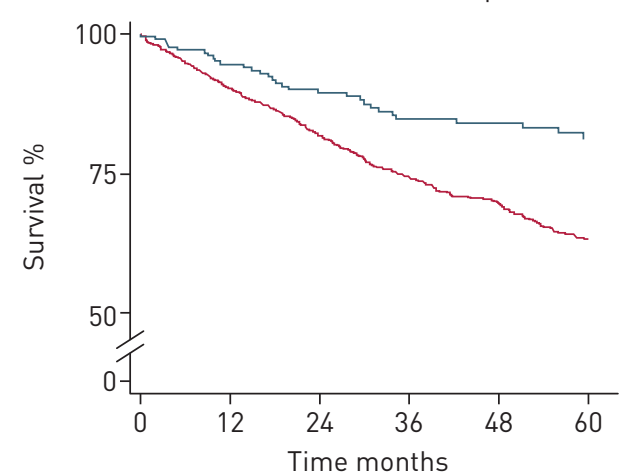

Patients $n$

$\begin{array}{lcccccc}\text { Non-AA } & 1398 & 986 & 706 & 509 & 389 & 283 \\ \text { AA } & 222 & 177 & 142 & 119 & 109 & 86\end{array}$

c)

$H R(95 \% C l) 0.6(0.3-1.1) ; p=0.086$

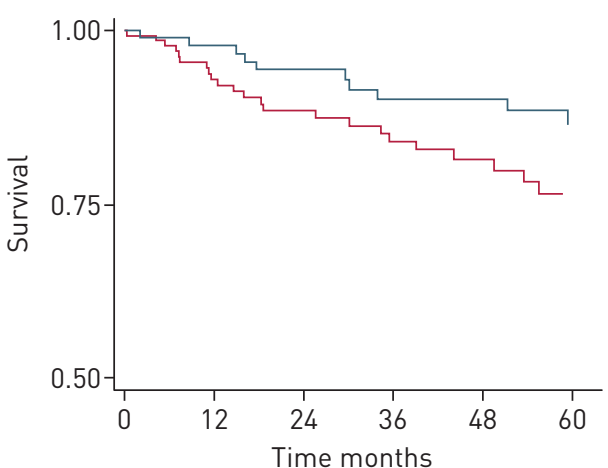

Patients $\mathrm{n}$

$\begin{array}{lllllll}\text { Non-AA } & 159 & 111 & 87 & 72 & 54 & 41 \\ \text { AA } & 104 & 86 & 72 & 62 & 59 & 46\end{array}$

e)

$\operatorname{HR}(95 \% \mathrm{CI})<0.1$;

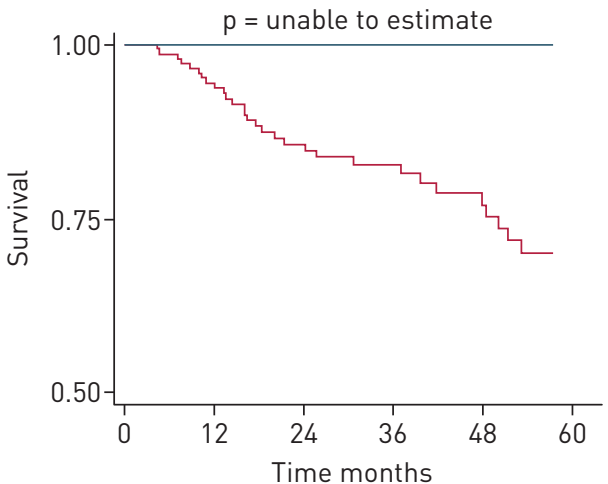

b)

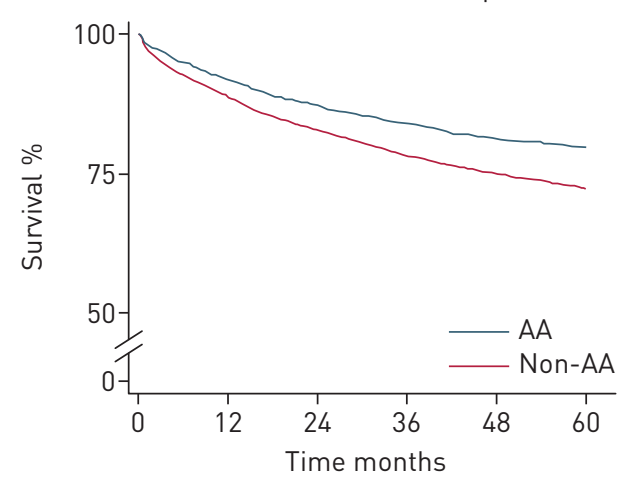

Patients $n$

$\begin{array}{lllllll}\text { Non-AA } & 6831 & 5763 & 4800 & 3987 & 3217 & 2455\end{array}$

$\begin{array}{llllllll}\text { AA } & 2540 & 2273 & 1954 & 1685 & 1461 & 1275\end{array}$

d)

$\operatorname{HR}(95 \% \mathrm{CI}) 0.2(0.1-0.7) ; p=0.008$

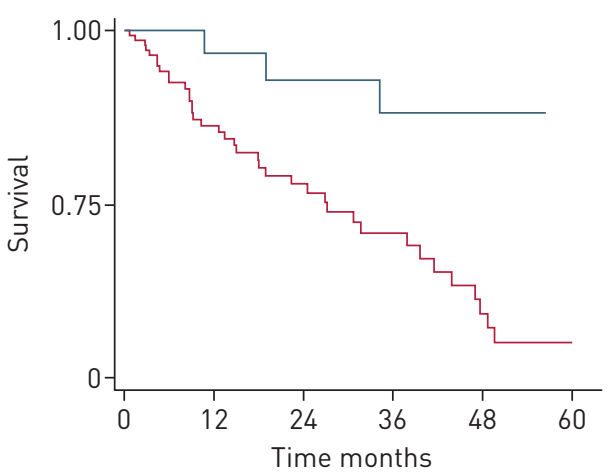

Patients $\mathrm{n}$

$\begin{array}{lllllll}\text { Non-AA } & 146 & 94 & 60 & 40 & 29 & 21\end{array}$

$\begin{array}{lllllll}\text { AA } & 34 & 29 & 23 & 18 & 16 & 13\end{array}$

f)

$\operatorname{HR}(95 \% \mathrm{CI}) 0.9(0.6-1.4) ; p=0.672$

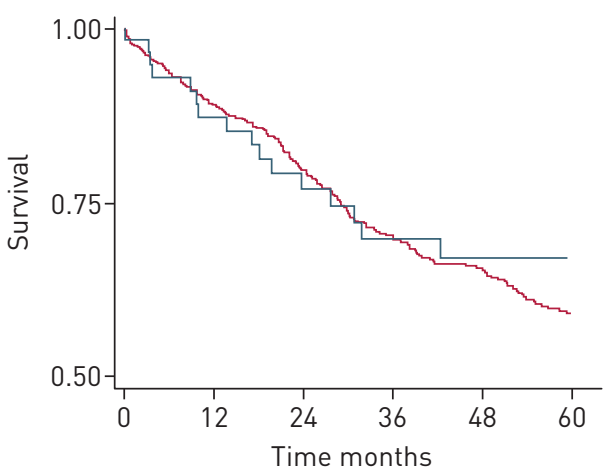

Patients $\mathrm{n}$

$\begin{array}{lllllll}\text { Non-AA } & 861 & 586 & 413 & 290 & 226 & 165\end{array}$

$\begin{array}{lcccccclcccccc}\text { Non-AA } & 152 & 130 & 95 & 68 & 45 & 37 & \text { Non-AA } & 861 & 586 & 413 & 290 & 226 & 165 \\ \text { AA } & 8 & 8 & 5 & 5 & 5 & 4 & \text { AA } & 63 & 44 & 34 & 26 & 21 & 18\end{array}$

FIGURE 2 Survival stratified by race among patients with a) multidisciplinary-diagnosed and adjudicated diagnosis of interstitial lung disease (ILD); b) code-based ILD diagnosis (consists of subjects with International Statistical Classification of Diseases, 9th revision based ILD diagnosis codes, excluding all patients with multidisciplinary or adjudicated ILD diagnosis); c) connective tissue disease-associated ILD d) interstitial pneumonia with autoimmune features; e) chronic hypersensitivity pneumonitis; f) idiopathic pulmonary fibrosis (IPF). Patients with unclassifiable diagnoses were included in the IPF category. AA: African-American; time zero (0): initial ILD evaluation at study centre. 
TABLE 3 Comparison of select baseline variables between African-American patients and non-African-American patients in the original (unmatched) and the matched interstitial lung disease (ILD) datasets

\begin{tabular}{|c|c|c|c|c|c|c|c|}
\hline & \multicolumn{2}{|c|}{ Original (unmatched) data } & \multirow[t]{2}{*}{ p-value } & \multicolumn{2}{|c|}{ Matched data } & \multirow{2}{*}{$\begin{array}{l}\text { Standardised } \\
\text { difference }{ }^{\#}\end{array}$} & \multirow[t]{2}{*}{ p-value } \\
\hline & $\begin{array}{l}\text { African-American } \\
\text { patients }\end{array}$ & $\begin{array}{c}\text { Non-African- } \\
\text { American } \\
\text { patients }\end{array}$ & & $\begin{array}{l}\text { African-American } \\
\text { patients }\end{array}$ & $\begin{array}{c}\text { Non-African- } \\
\text { American } \\
\text { patients }\end{array}$ & & \\
\hline Patients $\mathrm{n}$ & 222 & 1418 & & 180 & 126 & & \\
\hline Age years & $57.5 \pm 13.2$ & $67.8 \pm 11.9$ & $<0.001$ & $56.6 \pm 12.6$ & $57.8 \pm 13.1$ & 0.087 & 0.408 \\
\hline Male & $56(25.2)$ & $765(54.0)$ & $<0.001$ & $44(24.4)$ & $37(29.4)$ & 0.052 & 0.337 \\
\hline \multicolumn{8}{|l|}{ Disease severity index } \\
\hline GAP-ILD score & $4.2 \pm 1.7$ & $4.2 \pm 1.7$ & 0.802 & $4.3 \pm 1.7$ & $4.2 \pm 1.8$ & 0.023 & 0.683 \\
\hline \multicolumn{8}{|l|}{ Socioeconomic factors } \\
\hline Out-of-state referral & $23(10.4)$ & $221(15.6)$ & 0.042 & $22(12.2)$ & 15 (11.9) & $<0.001$ & 0.933 \\
\hline $\begin{array}{l}\text { Income level below } \\
\text { national median }\end{array}$ & $161(72.9)$ & 501 (35.5) & $<0.001$ & $134(74.4)$ & $87(69.1)$ & 0.039 & 0.300 \\
\hline $\begin{array}{l}\text { Medicare with private } \\
\text { insurance }\end{array}$ & $99(45.6)$ & 462 (33.3) & $<0.001$ & $82(45.6)$ & $57(45.2)$ & 0.022 & 0.956 \\
\hline
\end{tabular}

Data are presented as mean \pm SD or $\mathrm{n}(\%)$, unless otherwise stated. BMI: body mass index; GAP: gender-age-physiology. \#: comparing the frequency in the matched cohort to the unmatched cohort; " : forced vital capacity, diffusing capacity of the lungs for carbon monoxide, ILD subtype.

\section{Respiratory hospitalisation}

Respiratory hospitalisations constituted 50\% ( $\mathrm{n}=345)$ of all hospitalisations. Respiratory hospitalisations per patient (2.1 versus $1.8 ; \mathrm{p}=0.306)$ and risk for respiratory hospitalisation (RR 1.04, 95\% CI 0.86-1.27; $\mathrm{p}=0.658$ ) were similar between African-American and non-African-American patients (table 2). Similar findings were observed after race- and sex-specific cohort sub-classification (figure 4a). However, African-Americans hospitalised for respiratory causes had decreased mortality risk when compared to others (figure $4 \mathrm{~b}$ ).

\section{Discussion}

We have shown in a large, well-characterised multicentre cohort with diverse forms of ILD that African-American race is associated with 1) younger age at ILD diagnosis; 2) decreased all-cause mortality; 3) decreased risk for all-cause hospitalisation; and 4) similar rates of respiratory hospitalisation in patients with ILD. This novel study illuminates distinct clinical features and outcomes that characterise African-Americans with ILD while providing new insights into understanding disease aetiopathogenesis. Results of this study have implications for both ILD clinical care and research.

TABLE 4 Propensity score-matched model demonstrating association between African-American race and mortality

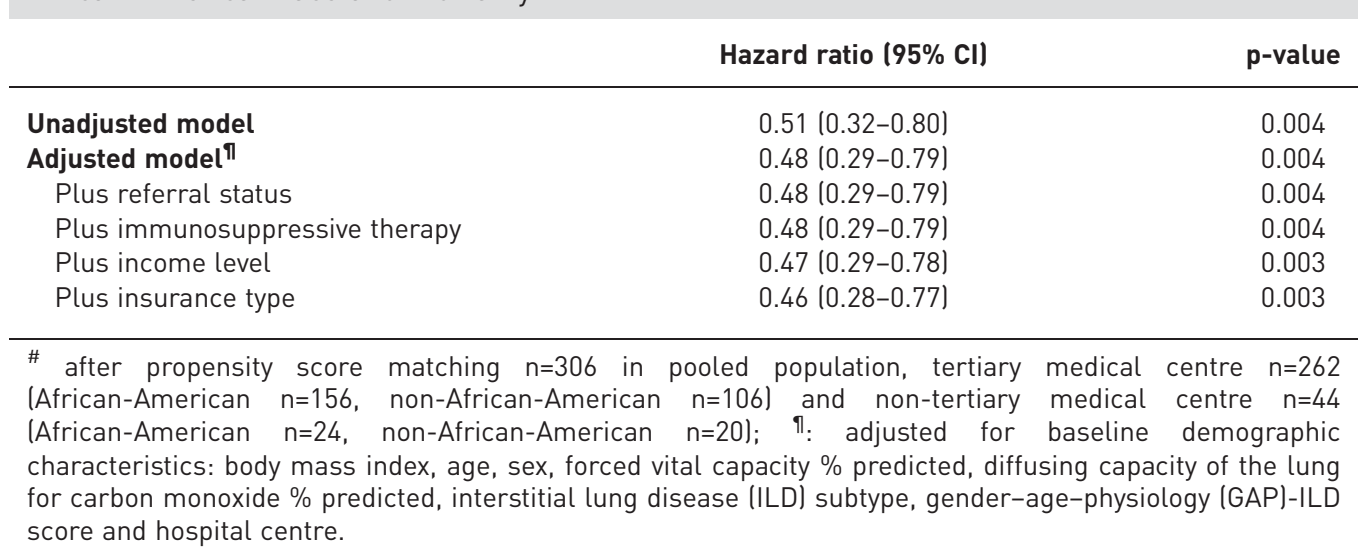


a)

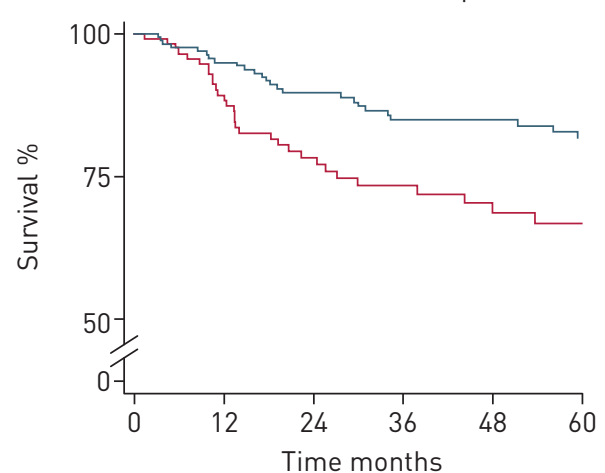

b)

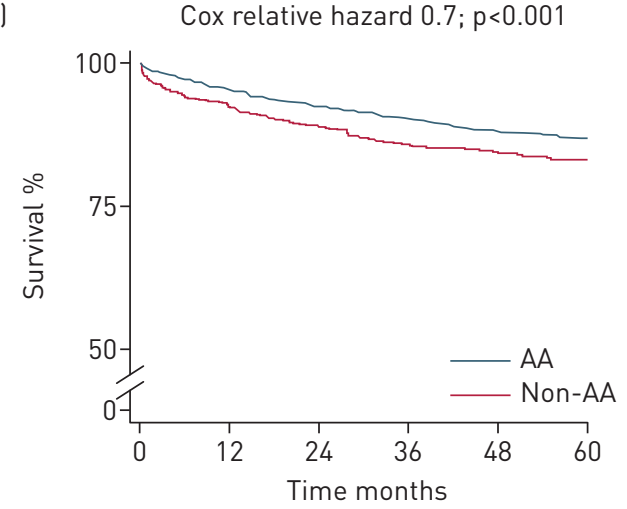

Patients $\mathrm{n}$

$\begin{array}{lllllll}\text { Non-AA } & 126 & 96 & 67 & 49 & 41 & 34\end{array}$

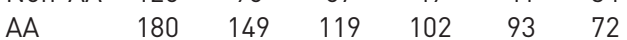

Patients $\mathrm{n}$

Non-AA $\quad \begin{array}{llllll}772 & 677 & 545 & 436 & 344 & 242\end{array}$

$\begin{array}{lllllll}\text { AA } & 1683 & 1552 & 1324 & 1125 & 947 & 798\end{array}$

FIGURE 3 Survival stratified by race among the propensity-matched cohorts with a) multidisciplinary and adjudicated diagnosis of interstitial lung disease (ILD); and b) code-based ILD diagnosis (consists of subjects with International Statistical Classification of Diseases, 9th revision based ILD diagnosis codes, excluding all patients with multidisciplinary or adjudicated ILD diagnosis). AA: African-American; time zero (0): initial ILD evaluation at study centre.

The primary outcome of improved all-cause mortality among African-Americans with ILD in our study remained consistent across tertiary and non-tertiary healthcare centres, and increased diagnostic specificity by replacing code-based diagnoses with MDD-ILD or adjudication of ILD diagnosis appeared to magnify this effect. However, although we adjusted for centre-level effects in our analyses, the inability to account for symptom duration before initial evaluation, the potential for subsequent referral from non-tertiary healthcare centres to other tertiary ILD centres and the low prevalence of African-American patients at these centres, all preclude broad conclusions about this observation.

Poor clinical outcomes in patients of African-American race are a unique public health challenge, which has been linked to socioeconomic disparities in access to care. As African-Americans and Caucasians receive care disproportionately at different centres, we selected several medical centres at divergent geographical locations, which distinctly differed in the prevalence of their African-American patient populations. In addition to socioeconomic factors, the high rates of respiratory impairment and decreased pulmonary function indices among African-Americans are well-described factors contributing to disparities in healthcare. Therefore, we derived propensity models attempting to capture several elements that enabled assessment of the independent effects of race. Our statistical models by which we matched on the propensity to be African-American accounted for demographic and pulmonary indices, while further adjusting for socioeconomic status, geographical location, access to care and immunosuppression use. The consistently improved survival in African-Americans across these models despite similar rates of respiratory hospitalisation suggests the effect of intrinsic patient-specific factors that transcend socioeconomic differences and use of immunosuppressive therapy.

Although epidemiological studies link mortality risk to geographical variations in disease prevalence, the observed prevalence of CTD-ILD among African-Americans in our study is consistent with that previously described by others $[26,27]$. The odds ratio for a diagnosis of autoimmune-associated forms of ILD among African-Americans in our cohort (CTD-ILD OR 5.9) was similar to that of patients of African descent with CTD/vasculitis ILDs in a recent French study (Afro-Caribbeans OR 4.4) [27]. The relatively low frequency of IPF observed among African-Americans in our cohort (OR 0.3) is similar to that of Swigris et al. [16], whose study demonstrated that African-Americans were significantly less likely to carry an ICD-9 diagnosis of IPF (OR 0.5). In addition, our findings are consistent with the ASCEND and CAPACITY trials, in which African-American patients constituted $<9 \%$ of the IPF population $[28,29]$. Similar to previous studies in IPF and scleroderma, African-Americans in our ILD cohort presented at an earlier age and had reduced lung function [13, 26, 30]. Yet, African-Americans with CTD-ILD in our cohort had a significant survival benefit that may have been associated with factors that have been described to improve survival in CTD-ILD, such as reduced prevalence of pulmonary fibrosis at presentation, differential autoantibody profiles or shared genetic variants [30-32].

DURHEIM et al. [33] showed that all-cause hospitalisations and respiratory hospitalisations predict survival in patients with IPF. Similarly, our findings demonstrate increased mortality in ILD patients with 
FIGURE 4 a) Percentage distribution of respiratory hospitalisations in the multidisciplinary and adjudicated interstitial lung disease (ILD) cohort stratified by race and sex; b) predictive margin interaction model demonstrating the relative mortality hazard in patients with multidisciplinary and adjudicated ILD diagnosis and respiratory hospitalisation. $\mathrm{n}=1640$.
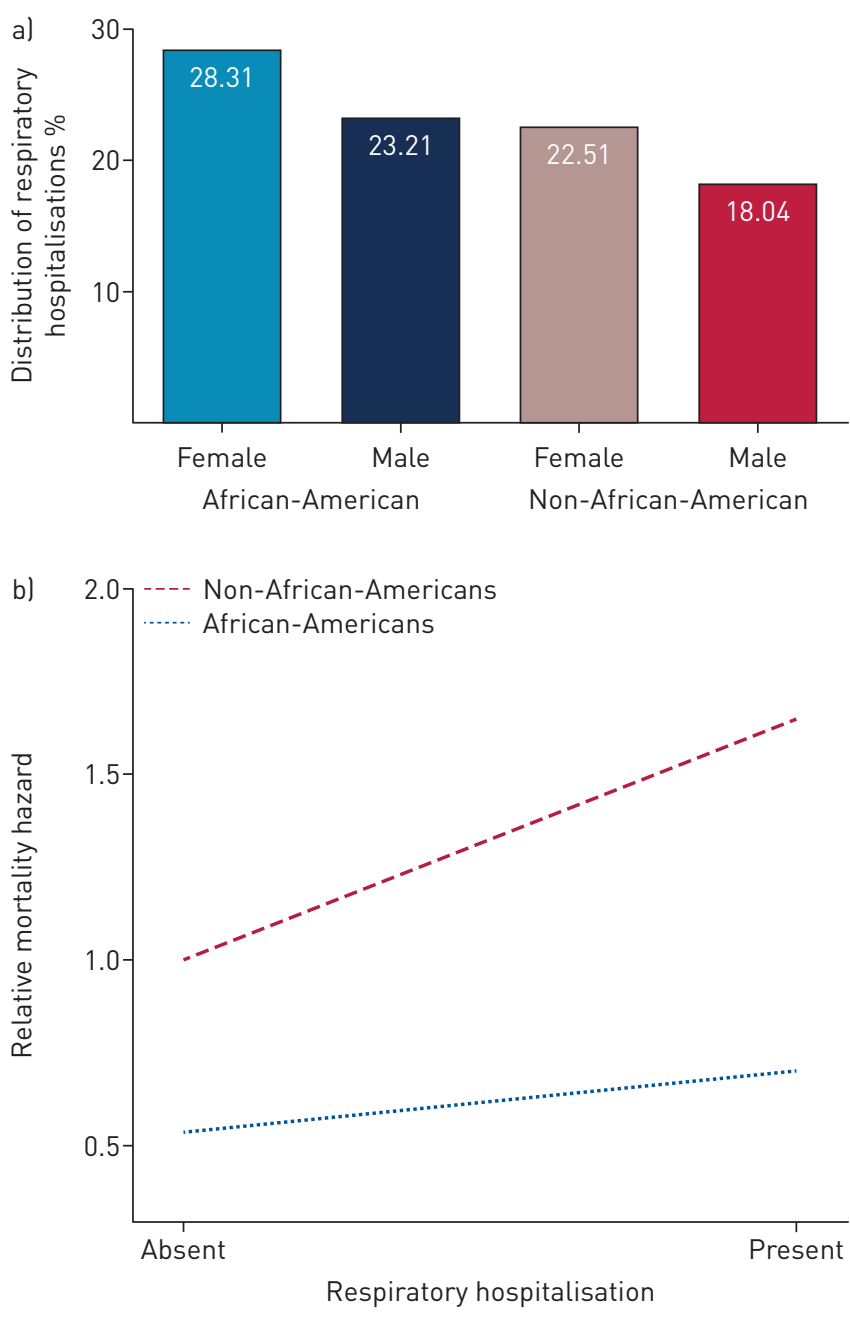

\begin{tabular}{lccc}
\hline & \multicolumn{3}{c}{ Predictive margin model } \\
\cline { 2 - 4 } Independent variables & Hazard ratio & $(95 \% \mathrm{CI})$ & $\mathrm{p}$-value \\
\hline African-American & 0.54 & $(0.32-0.75)$ & $<0.001$ \\
Respiratory hospitalisation & 1.65 & $(1.29-2.00)$ & $<0.001$ \\
Interaction & 0.70 & $(0.33-1.07)$ & $<0.001$
\end{tabular}

respiratory hospitalisation; however, this detrimental effect was less severe in African-American patients (figure 3b). Interestingly, the association of African-American race with improved survival despite comparable rates of respiratory hospitalisations in our study is similar to that from a recent post hoc analysis of patients with acute respiratory distress syndrome by JolLeY et al. [34], in which African-Americans who received conservative fluid management had better survival. This might suggest that the survival benefit observed in African-Americans with ILD occurs in those hospitalised for respiratory causes, or could be indicative of a unique African-American phenotype with distinct baseline characteristics which confer a survival advantage in advanced lung disease and should be fully explored in larger prospective studies.

Our study has several limitations. First, we excluded patients with sarcoidosis from our analysis as our study was designed to evaluate ILDs and the majority of patients with sarcoidosis have early-stage disease and lack parenchymal lung involvement. Second, patient identification at all study centres was performed retrospectively using code-based ILD diagnoses, which have been described to have a low diagnostic specificity [35]. However, we focused our analysis on the subset with a MDD-ILD and performed independent adjudication of diagnoses for all ILD patients from the non-tertiary centres. Due to the challenges of making an accurate assessment of specific ILD subtypes from heterogeneous data acquired retrospectively over a decade at these non-tertiary centres, the adjudication process was limited to diagnosing the presence of ILD in study participants, but not determining ILD subtypes at these centres. 
Therefore, the original ILD subtype diagnosis made by the local pulmonologist was utilised. Third, the balancing achieved by our propensity scoring was only performed on identified confounders rather than all possible confounders. Thus, some of the results of our study could be attributable to unmeasured confounding, even with the use of propensity scores, which are often used to measure treatment effects in observational studies. Fourth, race is a complex sociobiological construct that often reflects the patients' perception of their familial origin, cultural environment and genetic makeup. We verified ethnic identification at initial pulmonary evaluation using independent documentation of race by clinicians at the time pulmonary function tests were performed. Fifth, all hospitals in our study were located in the Greater Chicagoland area and our results could have been influenced by unmeasured environmental or geographic factors despite adjusting for residential location in our propensity score analyses. Future studies analysing a distinct cohort are needed.

\section{Conclusion}

ILD in adult African-American patients is characterised by a unique phenotype with diagnosis at a younger age, but with improved survival time when compared to non-African-Americans. This survival disparity may be influenced by familial, immunological or other biological mechanisms and the underlying potential pathways require further investigation.

Acknowledgments: Our profound appreciation goes to the support staff of the University of Chicago Respiratory Clinical Research Unit, the Interstitial Lung Disease Clinic (Nancy Trojan, Catherine Brown and Spring Maleckar) and NorthShore University HealthSystems (Evanston, IL, USA) (Mohammad Imran Beig, Daisy Zhu and Naomi BenIsrael Olive), for their assistance with this study. We also extend our gratitude to the patients with ILD who made these research endeavours possible.

Author's contributions: Conception and design: A. Adegunsoye, J.M. Oldham, I. Noth, M.E. Strek and M.M. Churpek. Acquisition of data for the work: A. Adegunsoye, J.M. Oldham, S.K. Bellam, J.H. Chung, P.A. Chung, K.M. Biblowitz, S. Montner, C. Lee, S. Hsu, A.N. Husain, R. Vij, G. Mutlu, I. Noth, M.M. Churpek and M.E. Strek. Analysis and interpretation: A. Adegunsoye, J.M. Oldham, I. Noth, M.E. Strek and M.M. Churpek. Drafting the manuscript for important intellectual content: A. Adegunsoye, J.M. Oldham, S.K. Bellam, J.H. Chung, P.A. Chung, K.M. Biblowitz, S. Montner, C. Lee, S. Hsu, A.N. Husain, R. Vij, G. Mutlu, I. Noth, M.M. Churpek and M.E. Strek. Critical revision for important intellectual content: all authors. Final approval of the submitted manuscript and accountability for all aspects of the work: all authors.

Conflict of interest: R. Vij received a grant from Genentech to study the genomics of autoimmune interstitial lung diseases. I. Noth received honoraria for advisory boards from Boehringer Ingelheim, InterMune and Anthera within the last 12 months related to IPF. He has also received speaking honoraria from GSK and consulting fees for Immuneworks. $\mathrm{He}$ also has study contracts with the NIH, Stromedix, Sanofi and BI for the conduct of clinical trials in IPF. M.M. Churpek is supported by a career development award from the National Heart, Lung, and Blood Institute (K08 HL121080), has received honoraria from Chest for invited speaking engagements and also has a patent pending (ARCD. P0535US.P2) for risk stratification algorithms for hospitalised patients. M.E Strek received institutional funding for interstitial lung disease research from Genentech, Gilead and MedImmune, and serves on a data monitoring committee for Boehringer Ingelheim. J.M. Oldham received speaking and advisory board fees from Genentech and Boehringer Ingelheim.

Support statement: This investigation was supported by a National Institutes of Health grant (T32-HL007605). Funding information for this article has been deposited with the Crossref Funder Registry.

\section{References}

1 American Thoracic Society, European Respiratory Society. American Thoracic Society/European Respiratory Society International Multidisciplinary Consensus Classification of the Idiopathic Interstitial Pneumonias. Am J Respir Crit Care Med 2002; 165: 277-304.

2 Raghu G, Chen SY, Yeh WS, et al. Idiopathic pulmonary fibrosis in US Medicare beneficiaries aged 65 years and older: incidence, prevalence, and survival, 2001-11. Lancet Respir Med 2014; 2: 566-572.

3 Hutchinson JP, McKeever TM, Fogarty AW, et al. Increasing global mortality from idiopathic pulmonary fibrosis in the twenty-first century. Ann Am Thorac Soc 2014; 11: 1176-1185.

4 Dressler WW, Oths KS, Gravlee CC. Race and ethnicity in public health research: models to explain health disparities. Annu Rev Anthropol 2005; 34: 231-252.

5 Schraufnagel DE, Blasi F, Kraft M, et al. An official American Thoracic Society/European Respiratory Society policy statement: disparities in respiratory health. Am J Respir Crit Care Med 2013; 188: 865-871.

6 Centers for Disease Control and Prevention. QuickStats: asthma death rates, by age and race group - United States, 2007-2009. MMWR 2012; 61: 315.

7 Vaz Fragoso CA, McAvay G, Gill TM, et al. Ethnic differences in respiratory impairment. Thorax 2014; 69: 55-62.

8 Cooke CR, Erickson SE, Eisner MD, et al. Trends in the incidence of noncardiogenic acute respiratory failure: the role of race. Crit Care Med 2012; 40: 1532-1538.

9 Heron M, Hoyert DL, Murphy SL, et al. Deaths: final data for 2006. Natl Vital Stat Rep 2009; 57: 1-134.

10 Haiman CA, Stram DO, Wilkens LR, et al. Ethnic and racial differences in the smoking-related risk of lung cancer. $N$ Engl J Med 2006; 354: 333-342.

11 Dransfield MT, Davis JJ, Gerald LB, et al. Racial and gender differences in susceptibility to tobacco smoke among patients with chronic obstructive pulmonary disease. Respir Med 2006; 100: 1110-1116. 
12 Foreman MG, Zhang L, Murphy J, et al. Early-onset chronic obstructive pulmonary disease is associated with female sex, maternal factors, and African American race in the COPDGene Study. Am J Respir Crit Care Med 2011; 184: 414-420.

13 McNearney TA, Reveille JD, Fischbach M, et al. Pulmonary involvement in systemic sclerosis: associations with genetic, serologic, sociodemographic, and behavioral factors. Arthritis Rheum 2007; 57: 318-326.

14 Mittoo S, Gelber AC, Christopher-Stine L, et al. Ascertainment of collagen vascular disease in patients presenting with interstitial lung disease. Respir Med 2009; 103: 1152-1158.

15 Goodwin A, Jenkins G. Idiopathic pulmonary fibrosis occurs more frequently in Caucasian than in Black patients - a systematic review. Am J Respir Crit Care Med 2015; 191: A1562.

16 Swigris JJ, Olson AL, Huie TJ, et al. Ethnic and racial differences in the presence of idiopathic pulmonary fibrosis at death. Respir Med 2012; 106: 588-593.

17 Kropski JA, Blackwell TS, Loyd JE. The genetic basis of idiopathic pulmonary fibrosis. Eur Respir J 2015; 45: 1717-1727.

18 Zamora-Legoff JA, Krause ML, Crowson CS, et al. Patterns of interstitial lung disease and mortality in rheumatoid arthritis. Rheumatology 2017; 56: 344-350.

19 Arias E, Heron M, Xu J. United States life tables, 2014. Natl Vital Stat Rep 2017; 66: 1-64.

20 Xian Y, Holloway RG, Noyes K, et al. Racial differences in mortality among patients with acute ischemic stroke: an observational study. Ann Intern Med 2011; 154: 152-159.

21 Spertus JA, Jones PG, Masoudi FA, et al. Factors associated with racial differences in myocardial infarction outcomes. Ann Intern Med 2009; 150: 314-324.

22 Silber JH, Rosenbaum PR, Clark AS, et al. Characteristics associated with differences in survival among black and white women with breast cancer. JAMA 2013; 310: 389-397.

23 Ryerson CJ, Vittinghoff E, Ley B, et al. Predicting survival across chronic interstitial lung disease: the ILD-GAP model. Chest 2014; 145: 723-728.

24 StataCorp. Stata Statistical Software: Release 15. College Station, TX, StataCorp SE, 2017.

25 Garrido MM, Kelley AS, Paris J, et al. Methods for constructing and assessing propensity scores. Health Serv Res 2014; 49: 1701-1720.

26 Gelber AC, Manno RL, Shah AA, et al. Race and association with disease manifestations and mortality in scleroderma: a 20-year experience at the Johns Hopkins Scleroderma Center and review of the literature. Medicine 2013; 92: 191-205.

27 Duchemann B, Annesi-Maesano I, de Naurois CJ, et al. Prevalence and incidence of interstitial lung diseases in a multi-ethnic county of Greater Paris. Eur Respir J 2017; 50: 1602419.

28 King TE Jr, Bradford WZ, Castro-Bernardini S, et al. A phase 3 trial of pirfenidone in patients with idiopathic pulmonary fibrosis. N Engl J Med 2014; 370: 2083-2092.

29 Noble PW, Albera C, Bradford WZ, et al. Pirfenidone in patients with idiopathic pulmonary fibrosis (CAPACITY): two randomised trials. Lancet 2011; 377: 1760-1769.

30 Steen V, Domsic RT, Lucas M, et al. A clinical and serologic comparison of African American and Caucasian patients with systemic sclerosis. Arthritis Rheum 2012; 64: 2986-2994.

31 Solomon JJ, Ryu JH, Tazelaar HD, et al. Fibrosing interstitial pneumonia predicts survival in patients with rheumatoid arthritis-associated interstitial lung disease (RA-ILD). Respir Med 2013; 107: 1247-1252.

32 Juge PA, Borie R, Kannengiesser C, et al. Shared genetic predisposition in rheumatoid arthritis-interstitial lung disease and familial pulmonary fibrosis. Eur Respir J 2017; 49: 1602314.

33 Durheim MT, Collard HR, Roberts RS, et al. Association of hospital admission and forced vital capacity endpoints with survival in patients with idiopathic pulmonary fibrosis: analysis of a pooled cohort from three clinical trials. Lancet Respir Med 2015; 3: 388-396.

34 Jolley SE, Hough CL, Clermont G, et al. Relationship between race and the effect of fluids on long-term mortality after acute respiratory distress syndrome. Secondary analysis of the Naional Heart, Lung, and Blood Institute Fluid and Catheter Treatment Trial. Ann Am Thorac Soc 2017; 14: 1443-1449.

35 Ley B, Urbania T, Husson G, et al. Code-based diagnostic algorithms for idiopathic pulmonary fibrosis. case validation and improvement. Ann Am Thorac Soc 2017; 14: 880-887. 